\title{
What is behind programmatic treatment outcome definitions for tuberculosis?
}

\author{
Zaza Avaliani ${ }^{1}$, Ogtay Gozalov², Giorgi Kuchukhidze ${ }^{2}$, Alena Skrahina ${ }^{3}$, \\ Viorel Soltan ${ }^{4}$, Martin van den Boom², Irina Vasilyeva ${ }^{5}$, Valentina Vilc ${ }^{6}$ and \\ Askar Yedilbayev ${ }^{2}$
}

Affiliations: ${ }^{1}$ National Center for Tuberculosis and Lung Diseases, Tbilisi, Georgia. ${ }^{2}$ WHO Regional Office for Europe, Copenhagen, Denmark. ${ }^{3}$ Republican Research and Practical Center for Pulmonology and Tuberculosis, Minsk, Belarus. ${ }^{4}$ Stop TB Partnership, Geneva, Switzerland. ${ }^{5}$ National Medical Research Center of Phthisiopulmonology and Infectious Diseases, Moscow, Russian Federation. 'Institute of Phthisiopneumology, Chisinau, Republic of Moldova.

Correspondence: Askar Yedilbayevb, WHO Regional Office for Europe, UN City, Marmorvej 51, DK-2100 Copenhagen, Denmark. E-mail: yedilbayevaAwho.int

Cite this article as: Avaliani Z, Gozalov O, Kuchukhidze G, et al. What is behind programmatic treatment outcome definitions for tuberculosis? Eur Respir J 2020; 56: 2001751 [https://doi.org/10.1183/ 13993003.01751-2020].

Following the launch of the DOTS (directly observed treatment, short-course) strategy in 1995, the World Health Organization (WHO) recommended the evaluation of treatment outcomes $[1,2]$. The methodology and the forms for recording and reporting, in order to programmatically evaluate the proportion of tuberculosis (TB) cases achieving treatment success ("cured" and "treatment completed"; table 1) or dying, failing treatment and being lost to follow-up, were initially developed by Karel Styblo in the 1980s as part of the pilot programme of TB control in Tanzania $[1,2]$.

The importance of collecting information and evaluating the TB control programmes using five simple outcomes has been reported previously [3]. It was developed within the Wolfheze Conferences [3], where the national TB experts representing the Member States of the WHO Europe Region meet every 2 years to set the policies and plans to fight TB in the region [3].

Presently, all national programmes are expected to use these end of treatment outcomes, which were last revised by WHO in 2013 separately for patients treated with regimens for rifampicin-susceptible TB and rifampicin-resistant TB [4].

Table 1 summarises the WHO definitions while commenting on their value and on possible future developments.

Recently, the possibility of modifying some of these definitions was discussed $[5,6]$.

This statement document aims to determine the core requirements of treatment outcome definitions to serve programmatic and clinical purposes and to avoid confusion with newly proposed definitions designed for different purposes. While historically multidrug-resistant (MDR)-TB outcome definitions were developed by the research community and adopted by programmes, we now need definitions for programmes which allow rapid evaluation and scale-up of treatment services. 
TABLE 1 Summary of the treatment outcome definitions for drug susceptible and rifampicin-resistant (RR), multidrug-resistant (MDR) and extensively drug-resistant (XDR) tuberculosis (TB)

\section{Outcome}

Drug-susceptible, isoniazid- and polydrug-resistant TB ${ }^{\#}$

Cured

Treatment completed

Failed

Died

Lost to follow-up

\section{Not evaluated}

RR/MDR/XDR-TB

A pulmonary TB patient with bacteriologically confirmed TB at the beginning of treatment who was smear- or month of treatment and on at least one previous occasion

A TB patient who completed treatment without evidence of failure BUT with no record to show that sputum smear or culture results in the last month of treatment and on at least one previous occasion were negative, either because tests were not done or because results are unavailable

A TB patient whose sputum smear or culture is positive at month 5 or later during treatment culture-negative in the last

Treatment completed as recommended by the national policy without evidence of failure AND three or more consecutive cultures taken at least 30 days apart are negative after the intensive phase

Treatment completed as recommended by the national policy without of failure BUT no record that three or more consecutive cultures taken at least 30 days apart are negative after the intensive phase

Treatment terminated or need for permanent regimen change of at least two anti-TB drugs because of:

- Lack of conversion by the end of the intensive phase", or

- Bacteriological reversion ${ }^{+}$in the continuation phase after conversion to negative, or

- Evidence of additional acquired resistance to fluoroquinolones or second-line injectable drugs, or

- Adverse drug reactions (ADRs)

A patient who dies for any reason during the course of treatment reason before or during the course of treatment

A TB patient who did not start treatment or whose treatment was interrupted for 2 consecutive months or more
A patient whose treatment was interrupted for 2 consecutive months or more
The definition might change in the future to capture the availability of rapid test for drug resistance and the change in the classification of anti-TB drugs lin which injectable drugs are not more considered crucial, while bedaquiline and linezolid have been recommended as priority drugs to use)

It may also be helpful for programmes to register a microbiological or clinical failure (non response) in which a change of regimen did not happen: clinicians may apply different thresholds to decide how many medicines to change

Additional sub-analyses including cases who died because of TB are possible in countries with capacities and resources

More granular information about monthly adherence or about prolongation of regimen due to sub-definitional interruptions may be helpful in future analysis for end of treatment outcome and true recrudescence post success
A patient for whom no treatment outcome is assigned (this includes cases "transferred out" to another treatment unit and whose treatment outcome is unknown)
A TB patient for whom no treatment outcome is assigned Ithis includes cases "transferred out" to another treatment unit as well as cases for whom the treatment outcome is unknown to the reporting unit) 
TABLE 1 Continued

Outcome
Drug-susceptible, isoniazid- and polydrug-resistant TB ${ }^{\#}$
RR/MDR/XDR-TB

The sum of cured and treatment completed
The sum of cured and treatment completed
Comments

\author{
This combined outcome is \\ necessary to allow cohort \\ analysis of both \\ bacteriologically positive and \\ negative pulmonary and \\ extrapulmonary cases
}

\footnotetext{
\#: outcomes for patients not on MDR/RR-TB treatment regimens. ": for treatment failed, lack of conversion by the end of the intensive phase implies that the patient does not convert within the maximum duration of intensive phase applied by the programme. If no maximum duration is defined, an 8-month cut-off is proposed. For regimens without a clear distinction between intensive and continuation phases, a cut-off 8 months after the start of treatment is suggested to determine when the criteria for cured, treatment completed and treatment failed start to apply. ": the terms "conversion" and "reversion" of culture as used here are defined as follows. Conversion (to negative): culture is considered to have converted to negative when two consecutive cultures, taken at least 30 days apart, are found to be negative. In such a case, the specimen collection date of the first negative culture is used as the date of conversion. Reversion (to positive): culture is considered to have reverted to positive when, after an initial conversion, two consecutive cultures, taken at least 30 days apart, are found to be positive. For the purpose of defining treatment failed, reversion is considered only when it occurs in the continuation phase. Modified from [4].
}

In order to serve best country- and setting-specific TB interventions, outcome definitions should be programme-based and meet the following criteria:

1) To include all registered patients, including those clinically diagnosed (e.g. not bacteriologically confirmed), children and extrapulmonary cases.

2) To be simple enough to allow use in countries still unable to benefit from ongoing surveillance based on individual computerised data [5]. They need also to ensure global comparability.

3) To allow quarterly and annual evaluation of the TB programme at both the peripheral and the national level, in order to make prompt public health decisions [5].

4) To be applicable prospectively and thus allow timely clinical action on individual patients [5].

Historically the treatment outcome definitions were initially based on sputum smear, rather than on culture, and, more recently, on bacteriological examinations including rapid diagnostic tests [4].

The WHO definition of cure was based on the bacteriological evidence of a negative sputum smear and/or culture for the patients being bacteriologically positive at diagnosis. Due to the complexity of managing rifampicin-resistant (RR)-TB, the introduction of more appropriate definitions based on the consistent evidence of sufficient negative cultures in the final part of treatment were necessary. The definition was simplified in 2013, with three negative cultures taken at least 30 days after the preceding one during the continuation phase of treatment [4] (table 1). It is important to note that the 2013 definitions were aimed at ensuring that "cure" and "failure" would be definable prospectively, and would not require a retrospective evaluation after termination of therapy. Additionally, the need of a larger number of negative cultures required in the continuation phase to define "cure" has the potential to result in prolongations of the "longer" individualised regimens.

In addition, the 2013 outcomes were introduced at a time when the prospect of programmatic use of the shorter treatment regimens began to appear imminent.

The outcome "cured" is sometimes difficult to evaluate, since patients being successfully treated include a relatively high proportion of non-sputum producers. For this reason, and to allow evaluation of bacteriologically negative patients, the outcome "treatment completed" was introduced. "Treatment success", e.g. the sum of "cured" and "treatment completed" is the combined outcome presently used to evaluate positive outcomes [4].

The WHO definition of failure for drug-susceptible TB was based on a persistent bacteriological positivity at month 5 [4].

In 2013, to capture the increasing complexity of RR-, MDR- and extensively drug-resistant (XDR)-TB, the definition was modified focusing on a significant change in the regimen (i.e. emphasising that it was the treatment that was failing and not the patient). The previous definition stated that "Treatment will be considered to have failed if two or more of the five cultures recorded in the final 12 months 
are positive, or if any one of the final three cultures is positive. Treatment will also be considered to have failed if a clinical decision has been made to terminate treatment early due to poor response or adverse events."

The criteria of the 2013 definition include lack of conversion by the end of the intensive phase, or bacteriological reversion in the continuation phase after conversion to negative, or evidence of additional acquired resistance to fluoroquinolones or second-line injectable drugs, or adverse drug reactions requiring discontinuation of at least two drugs $[4,5]$.

The definition of death includes death for any cause, to comply with the need to keep it as simple as possible $[4,5]$.

Recently, clinical and research-oriented definitions of cure and failure have been proposed based on the fact that some people who finish treatment without signs of failure will nonetheless relapse shortly after or die from sequelae. It was thus proposed to have a 1 year of follow-up for patients who are cured or who complete treatment successfully to assess disease-free survival $[6,7]$.

The year of post-treatment observation would allow a more careful assignment of some patients to a different outcome category. In particular, this approach would allow assignment of some of the patients relapsing (being previously treated and categorised as cured or treatment completed) to the category "failure".

It is important to underline that this approach, like others, has built-in advantages and disadvantages.

First, a/this definition cannot be evaluated correctly if all strains are not studied by fingerprinting to differentiate true relapses from re-infections, which are quite common in high TB burden settings [5]. We encourage this approach, where feasible.

In addition, the relevance of having 1-year follow-up and evaluating carefully the relapses (and eventually, deaths) would be that of evaluating for any resistance which might have developed during treatment and to start appropriate treatment as soon as possible [5]. No evidence is presently available on this aspect to support new definitions.

The approach of including follow-up is not new, having been used in controlled clinical trials since the 1950s. From the historical trials on streptomycin to the recent studies evaluating new drugs or the shorter MDR-TB regimen through quality observational studies, the approach of including 24-36 month follow-up was consistently used $[8,9]$. However, the purpose of these studies is to evaluate "a treatment regimen" and not a "TB programme."

The features of the research-oriented clinical definitions proposed recently do not sufficiently meet the basic requirements mentioned above. 1) They cannot be applied to all cases, as they are based on cultures. Although it is true that those patients are the ones more likely to transmit the infection, a programmatic definition should cover all the cases included in the National TB Register. 2) It is clearly not possible for the majority of countries with high burden and lower resources to follow-up their cases for 1 year following the end of treatment [5]. The amount of extra work is not currently feasible and the cost implications would not be manageable or be out of proportion of potential benefits, particularly in resource-limited countries. Furthermore, any change of existing definitions implies significant costs to modify the surveillance system: updating guidelines, printing new forms, training health staff, adapting surveillance software, etc. Any changes may also potentially hamper the reporting to WHO for its annual report, and pros and cons have hence to be very carefully weighted. Investment into surveillance is recommended, to ensure simple, complete and useful information are made available to support decision-making. 3) Any result made available 1 year after completing treatment (which is 2 years from the start of the calendar year of treatment for drug-susceptible TB cohorts and 3 years for MDR-TB cohorts) is too late to allow rapid public health action. 4) Clinical action on failures cannot be taken at this point in time. The definition has therefore no clinical value. However, if/when feasible a more precise assessment of failures might have programmatic value.

Clearly, the availability of quality data allows to be more precise. For example, it is true that several European countries can differentiate deaths due to TB from those due to other unrelated causes (e.g. a car accident). However, in order to ensure global data comparability the inclusive definition is necessary, although individual countries are welcome to undertake more precise sub-analyses.

In conclusion, no definition is perfect. Future WHO treatment outcome definitions will require taking into account faster detection of failure given the availability of new rapid diagnostic tests (and deceasing role of sputum smear microscopy), shortened duration of the newly recommended treatment regimens without clearly defined intensive and continuation phases, or new case definitions of XDR-TB aligned to the updated WHO classification of medicines for use in RR-TB regimens $[5,10]$. This will be done with input from TB programmes, implementers and technical partners. 
Research-oriented definitions, aimed at answering specific research questions or at evaluating treatment regimens are necessary for these purposes, which are different from the programmatic evaluation of national TB programmes. A framework for reporting of individual outcomes in patients treated for MDR/ RR-TB has recently been published and could help in improving future global policy on MDR-TB regimens, much of which remains reliant on observational data [11].

In the coming future, digital technologies will make it feasible to capture more data (including patients' identifiers) more reliably (e.g. looking to patients instead of treatment episodes) and monitor patient wellbeing and survival prospectively (e.g. as already done for cancer registries).

Nevertheless, the existing definitions to date represent a valid and standardised tool to evaluate national TB programmes. At this stage, they can be seen as ways of complementing one another, rather than one replacing the other, depending on the purpose they serve.

Conflict of interest: None declared.

\section{References}

Sotgiu G, Spanevello A, Migliori GB. History of tuberculosis and drug resistance. N Engl J Med 2013; 368: 88-89.

2 World Health Organization. Global Tuberculosis Report 2019. Geneva, World Health Organization, 2019. WHO/ $\mathrm{CDS} / \mathrm{TB} / 2019.15$

3 Veen J, Raviglione MC, Rieder HL, et al. Standardized tuberculosis treatment outcome monitoring in Europe: recommendations of a Working Group of the World Health Organization (WHO) and the Europe Region of the International Union against TB and Lung Disease (IUATLD) for uniform reporting by cohort analysis of treatment outcome in tuberculosis patients. Eur Respir J 1998; 12: 505-510.

4 World Health Organization. Definitions and Reporting Framework for Tuberculosis - 2013 Revision (updated December 2014 and January 2020). Geneva, World Health Organization, 2019. WHO/HTM/TB/2013.2

5 Migliori GB, Global Tuberculosis Network (GTN). Evolution of programmatic definitions used in tuberculosis prevention and care. Clin Infect Dis 2019; 68: 1787-1789.

6 Chesov D, Alexandru S, Crudu V, et al. Failing treatment of multidrug-resistant tuberculosis: a matter of definition. Int J Tuberc Lung Dis 2019; 23: 522-524.

7 Dedicoat MJ, Günther G, Crudu V, et al. Treatment outcomes in europe: based on treatment completion, not cure. Am J Respir Crit Care Med 2017; 196: 1222-1224.

8 Rieder HL. Epidemiologic Basis of Tuberculosis Control. 1stEd. Paris, International Union Against Tuberculosis and Lung Disease, 1999; pp. 1-162 Available from: www.theunion.org/what-we-do/publications/english/pub_ epidemiologic_basis_eng.pdf

9 Van Deun A, Maug AK, Salim MA, et al. Short, highly effective, and inexpensive standardized treatment of multidrug-resistant tuberculosis. Am J Respir Crit Care Med 2010; 182: 684-692.

10 Pontali E, Raviglione MC, Migliori GB, et al. Regimens to treat multidrug-resistant tuberculosis: past, present and future perspectives. Eur Respir Rev 2019; 28: 190035.

11 Campbell JR, Falzon D, Mirzayev F, et al. Improving quality of patient data for treatment of multidrug- or rifampin-resistant tuberculosis. Emerging Infect Dis 2020; 26: 10.3201/eid2603.190997. 